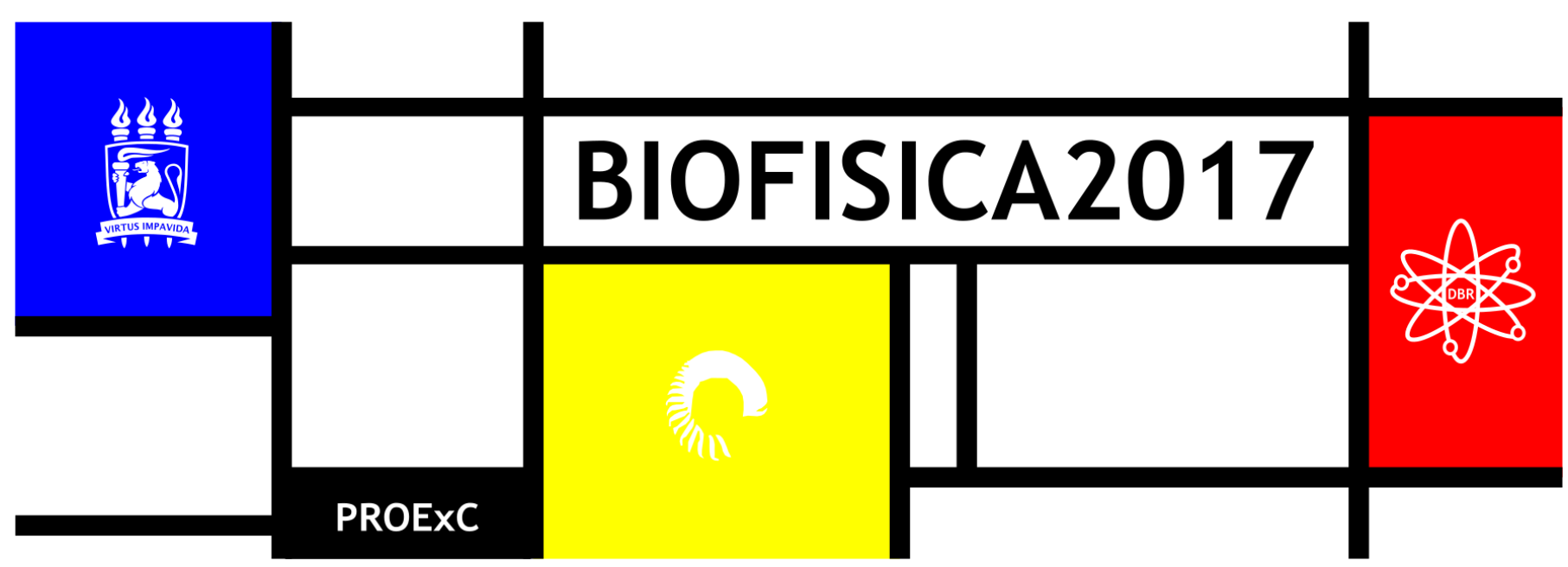

\title{
A BUSCA DE PADRÕES DE REFERÊNCIA PARA ESTUDOS AMBIENTAIS UTILIZANDO INVERTEBRADOS TERRESTRES
}

\author{
Evely Elen de Sousa ${ }^{1 *}$, Mariana Luiza de Oliveira Santos ${ }^{1}$, Marcelo da Rocha Leão Magalhães ${ }^{1}$, \\ Marina Cordeiro Barbosa ${ }^{1}$, Elvis Joacir de França ${ }^{1}$ \\ ${ }^{1}$ Centro Regional de Ciências Nucleares do Nordeste-CRCN/NE \\ *evelywizziac@hotmail.com
} \begin{abstract}
INTRODUÇÃO
A qualidade do ecossistema pode ser afetada significativamente a partir da alteração da disponibilidade de substâncias químicas para o ambiente. Como a acumulação dessas substâncias na teia alimentar é indiscutível, torna-se essencial o conhecimento das concentrações naturais de elementos químicos, seus possíveis efeitos na biota e incorporação de substâncias químicas por processos ecológicos (FONSECA, 2010).

Para facilitar a comparação com estudos ambientais em áreas impactadas, a escolha da região de referência é relevante, principalmente para áreas naturais protegidas, cujas interferências externas quanto à disponibilidade de elementos químicos podem ser consideradas reduzidas. O Estado de Pernambuco mantém apenas $2 \%$ da cobertura original em fragmentos inseridos em mosaicos por monoculturas e aglomerados urbanos
\end{abstract} (CHIARELLO, 1999, SILVA; TABARELLI et al., 2006).

Os invertebrados terrestres podem ser empregados em estudos de avaliação de qualidade ambiental, pois sua composição de elementos químicos reflete a qualidade de um ecossistema. (SCHOFIELD et al., 2002). Este grupo de organismos é bastante relevante ao considerar-se seu papel ecológico no ecossistema e na densidade populacional elevada.

Pesquisas envolvendo a busca por padrões de referência para estudos ambientais a partir de insetos necessitam das mais variadas técnicas analíticas para quantificação do maior número de elementos químicos possíveis e as suas respectivas concentrações. As técnicas analíticas multielementares de Fluorescência de Raios$X$ por Energia Dispersiva (EDXRF), Espectrometria de Absorção Atômica (AAS) e Espectrometria de Massas por Plasma Acoplado Indutivamente (ICP-MS) merecem destaque devido às suas respectivas multielementaridades, ou seja, determinação simultânea de diversos elementos químicos.

Esta pesquisa teve como objetivo avaliar as concentrações naturais de elementos químicos em invertebrados terrestres encontrados na Reserva Refúgio Ecológicos Charles Darwin, Reserva Particular do Patrimônio Natural (RPPN) localizada no Município de Igarassu,
Litoral Norte de Pernambuco que possam ser usadas como valores de referência para estudos ambientais em áreas urbanas e avaliação de impactos causados por atividades antropogênicas.

\section{MATERIAL E MÉTODOS}

O Refúgio Ecológico Charles Darwin caracteriza-se por ser uma área não impactada que pode ser utilizada para estudos ambientais, possui 60 ha e está localizado no Estado de Pernambuco, no município de lgarassu (coordenadas geográficas: latitude $7^{\circ} 48^{\prime} 51.34^{\prime \prime}$; ; longitude $\left.34^{\circ} 57^{\prime} 13.31^{\prime \prime} \mathrm{W}\right)$.

Os invertebrados terrestres utilizados neste trabalho foram coletados por meio de armadilhas do tipo "pitfall" (Fonseca, 2010) e lavados com água destilada para a retirada de material exógeno, em seguida, separados e identificados em nível de ordem com auxílio de lupa, utilizando atributos característicos das principais ordens de insetos. Amostra composta dos insetos coletados (de forma a conseguir massa razoável para os testes) foi congelada, e em seguida, liofilizada até peso constante (aproximadamente 48 horas).

Após a liofilização, a amostra foi cominuída com pistilo e almofariz de vidro para evitar contaminação com elementos químicos, até a obtenção de partículas de tamanho inferior a 0,5 mm. Foram amostrados cerca de 820 insetos das ordens Hymenoptera, Diptera, Coleoptera, Orthoptera e Blattodea para a realização do estudo de tratamento químico de amostras. As análises químicas das amostras de invertebrados terrestres seguem a metodologia aplicada por Magalhães (2015).

\section{RESULTADOS E DISCUSSÃO}

$\mathrm{Na}$ Tabela 1 estão apresentados as concentrações dos elementos químicos encontradas nos invertebrados terrestres do Refúgio Ecológico Charles Darwin. 
Tabela 1. Concentrações médias obtidas $(\mathrm{mg} / \mathrm{kg}$ ) para amostras de insetos por EDXRF, GFAAS e ICP-MS, e suas respectivas regiões de alta densidade de probabilidade - HPD em nível de $95 \%$ de confiança

\begin{tabular}{|c|c|c|c|c|c|c|}
\hline Analito & & As & Cd & $\mathrm{Cl}$ & $\mathrm{Cu}$ & $\mathrm{Fe}$ \\
\hline Média & & 0,24 & 0,23 & 1300 & 29 & 340 \\
\hline Regiões & $\min$ & 0,001 & 0,18 & 1000 & 12 & 230 \\
\hline HPD 95\% & $\max$ & 0,90 & 0,28 & 1800 & 45 & 460 \\
\hline$n$ & & 4 & 4 & 9 & 4 & 4 \\
\hline Analito & & $P$ & $\mathrm{~Pb}$ & $\mathrm{~s}$ & Sb & Th \\
\hline Média & & 8500 & 1,1 & 3400 & 0,026 & 0,25 \\
\hline Regiões & $\min$ & 7500 & 0,62 & 3100 & 0,010 & 0,14 \\
\hline HPD 95\% & $\max$ & 9700 & 1,5 & 3700 & 0,041 & 0,36 \\
\hline $\mathrm{n}$ & & 13 & 4 & 14 & 3 & 4 \\
\hline
\end{tabular}

Nas Figuras 1, 2 e 3 estão apresentadas as concentrações dos elementos químicos As, Cu e $\mathrm{Zn}$ determinadas em invertebrados terrestres de áreas urbanas e comparadas com concentrações de elementos químicos encontradas em invertebrados do Refúgio Ecológico estudado. 0 arsênio, considerado tóxico aos seres vivos, apresentou concentrações na faixa de valores de referência estabelecidos por Magalhães (2015) em todas as regiões estudadas. As concentrações de As foram semelhantes às de $\mathrm{Cu}$ na região do manguezal urbano do Parque Memorial Arcoverde, porém, para o Campus Tecnológico e a Mata do Frio, $\mathrm{Cu}$ apresentou tendência de teores abaixo dos valores padrão estabelecidos por Magalhães (2015). Trabalhos realizados por Talarico et al. (2014) em regiões poluídas com metais pesados, apresentaram acúmulo de $\mathrm{Cu}$ em concentrações de $10 \quad \mathrm{mgkg}^{-1}$ em Coleoptera. Karadjova e Markova (2009) encontraram cerca de $21 \mathrm{mg} \mathrm{kg}^{-1}$ a 40 $\mathrm{mg} \mathrm{kg}^{-1}$ deste metal em Orthoptera, indicando que a área do Refúgio Ecológico escolhida como referência pode estar sofrendo impacto com relação a esse elemento químico. Apenas os invertebrados das ordens Blattodea do Campus Tecnológico e Craterostigmomorpha da Mata do Frio estiveram dentro da faixa de concentrações de referência (MAGALHÃES, 2015).

Com relação a $\mathrm{Zn}$, foram obtidas concentrações acima dos valores de referência estabelecidos por Magalhães (2015) nas ordens Craterostigmomorpha na Mata do Frio e Diptera do manguezal Arcoverde. Mélo (2014), avaliando moluscos terrestres deste manguezal urbano, também encontrou concentrações elevadas de zinco nos espécimes coletados. Zinco é um elemento químico essencial aos organismos, estando presente em mais de setenta enzimas, responsável pelo metabolismo de carboidratos, proteínas, lipídeos e ácidos nucléicos (YU et al., 2014), todavia, quando em quantidades elevadas, pode ser tóxico aos indivíduos.

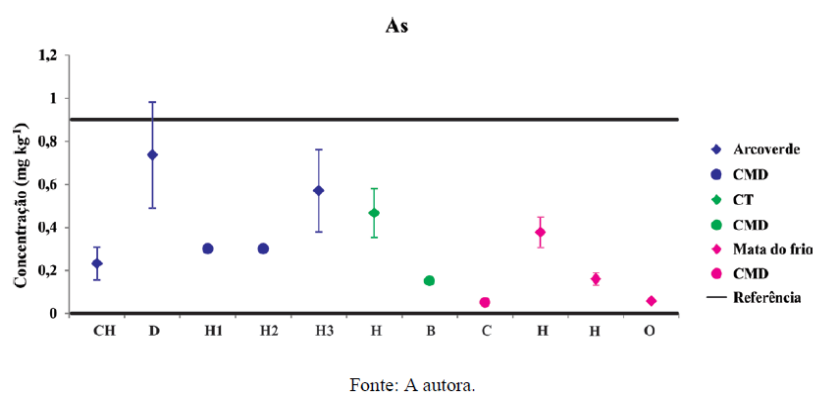

Figura 1 - Comparação entre as concentrações de As determinadas nas amostras de invertebrados terrestres e valores de padrões de referência de estudos ambientais

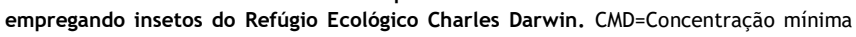
determinada.CT=Campus Tecnológico. $\mathrm{CH}=$ Composta Hymenoptera. $\mathrm{D}=$ Diptera. $\mathrm{H}=$ Hymenoptera. $\mathrm{B}=$ Blattodea. $\mathrm{C}=$ Craterostigmomorpha. $\mathrm{O}=$ Orthoptera.

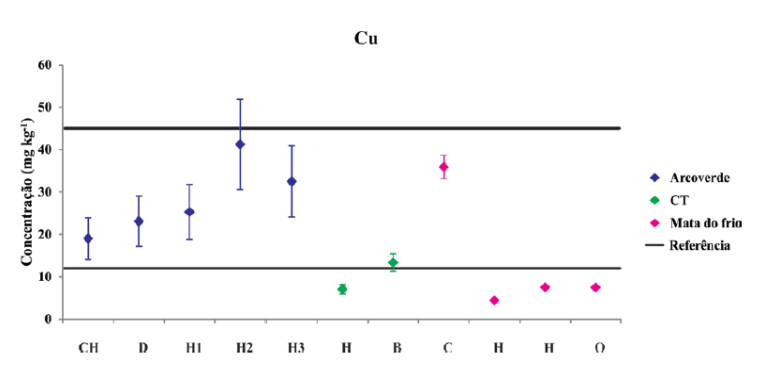

Figura 2 - Comparação entre as concentrações de Cu determinadas nas amostras de invertebrados terrestres e valores de padrões de referência de estudos ambientais empregando insetos do Refúgio Ecológico Charles Darwin. CMD=Concentração mínima determinada. $\mathrm{CT}=$ Campus Tecnológico. $\mathrm{CH}=$ Composta Hymenoptera. $\mathrm{D}=$ Diptera. $\mathrm{H}=$ Hymenoptera. $\mathrm{B}=$ Blattodea. $\mathrm{C}=$ Craterostigmomorpha. $\mathrm{O}=$ Orthoptera

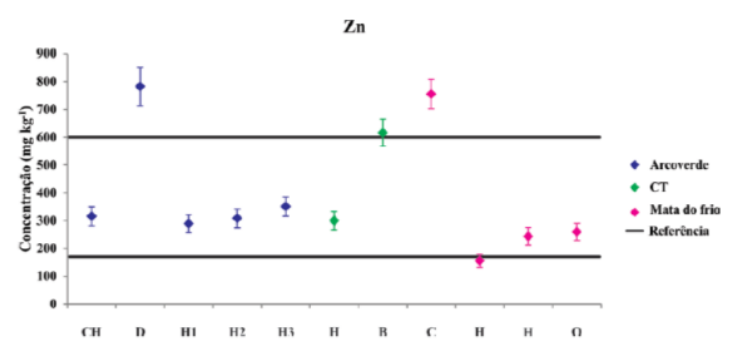

Figura 3 - Comparação entre as concentrações de Zn determinadas nas amostras de invertebrados terrestres e valores de padrões de referência de estudos ambientais empregando insetos do Refúgio Ecológico Charles Darwin. $C M D=$ Concentração mínima determinada. $\mathrm{CT}=$ Campus Tecnológico. $\mathrm{CH}=$ Composta Hymenoptera. $\mathrm{D}=$ Diptera $\mathrm{H}=$ Hymenoptera. $\mathrm{B}=$ Blattodea. $\mathrm{C}=$ Craterostigmomorpha. $\mathrm{O}=$ Orthoptera

$\mathrm{Na}$ Figura 4 estão representadas as concentrações obtidas dos elementos químicos Cd em invertebrados terrestres. Nos invertebrados do manguezal Arcoverde, as concentrações obtidas de $\mathrm{Cd}$ e $\mathrm{S}$ em amostras de Hymenoptera mostraram-se acima dos valores estimados como referência por Magalhães (2015). A faixa de concentração de Cd para invertebrados pode ter sido uma característica do Refúgio Ecológico Charles Darwin.

A concentração $0,3 \mathrm{mg} . \mathrm{kg}-1$ de Cd obtida de invertebrados está de acordo com a faixa de 0,03-0,5 mg.kg-1 estabelecida para plantas. Vale ressaltar que a preocupação com acúmulo deste elemento químico por organismos vivos merece atenção diante da toxicidade do $\mathrm{Cd}$, o qual é reconhecidamente prejudicial ao funcionamento de órgãos como rins e fígado em seres humanos (PAYLISS et al., 2015). Para o Campus Tecnológico, as concentrações obtidas de Cd, estiveram dentro dos valores de referência de Magalhães (2015), com exceção das amostras de Hymenoptera. Amostras de invertebrados das ordens Craterostigmomorpha e Orthoptera da Reserva Ecológica Mata do Frio apresentaram valores inferiores à faixa de concentração química estabelecida por Magalhães (2015) para o Cd.

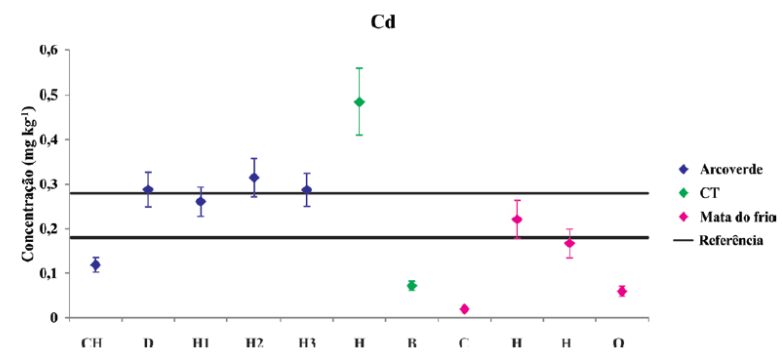

Figura 4 - Comparação entre as concentrações de Cd determinadas nas amostras de invertebrados terrestres e valores de padrões de referência de estudos ambientais

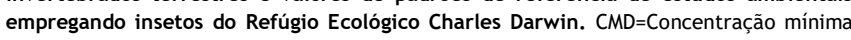
determinada.CT=Campus Tecnológico. CH=Composta Hymenoptera. D=Diptera. $\mathrm{H}=$ Hymenoptera. $\mathrm{B}=$ Blattodea $. \mathrm{C}=$ Craterostigmomorpha. $\mathrm{O}=$ Orthoptera 


\section{CONCLUSÕES}

A partir do emprego de invertebrados terrestres de áreas naturais para quantificar concentrações de elementos químicos e produzir valores de referência para estudos ambientais, ao se comparar com concentrações quantificadas em invertebrados de áreas urbanas, foi possível verificar que com a aplicação das técnicas analíticas GFAAS, EDXRF e ICP-MS, os elementos químicos As, Cd, Cu e Zn foram quantificados nos tecidos dos invertebrados terrestres pertencentes às ordens Hymenoptera, Orthoptera, Blattodea, Craterostigmomorpha e Diptera e que das áreas urbanas investigadas, o manguezal Parque Memorial Arcoverde apresentou maiores características de ambiente impactado, devido às maiores concentrações de elementos tóxicos como $\mathrm{Cd}$, Cu e $\mathrm{Zn}$.

\section{REFERÊNCIAS}

CHIARELLO, A. G. Effects of fragmentation of the Atlantic forest on mammal communities in southeastern Brazil. Biological Conservation, v. 89, p.71-82, 1999.

FONSECA F. Y. Aplicabilidade do compartimento ecológico fauna em estudos de avaliação da qualidade ambiental. Dissertação de Mestrado. Piracicaba: Centro de Energia Nuclear na Agricultura, Universidade de São Paulo, p. 107, 2010.

KARADJOVA, I.; MARKOVA, E. Metal accumulation in insects (Orthoptera, Acridae) near copper smeter and copper-flotation factory (Pirdop, Bulgaria). Biotechnology \& Biotechnological Equipment, v. 23, p. 204 - 207, 2009.

MAGALHÃES, M. R. L. Concentrações naturais de elementos químicos da classe insecta do fragmento florestal de mata atlântica reserva charles Darwin. Universidade Federal de Pernambuco. Dissertação (Mestrado), Tecnologias Energéticas e Nucleares, p. 1-99, 2015.

MÉLO, J. T. B. Moluscos Terrestres Littoraria angulifera e Melampus coffea como biomonitores da qualidade ambiental de Manguezais Pernambucanos. Universidade Federal de Pernambuco. Dissertação (Mestrado), Tecnologias Energéticas e Nucleares, p. 1-77, 2014.

SCHOFIELD, R. M .S.; NESSON, M. H.; RICHARDSON, K. A. Tooth hardness increases with zinc-content in mandibles of young adult leaf-cutter ants. Naturwissenschaften, v. 89, p. 579-583, 2002.

TABARELLI, M.; MELO, M. D. V. C.; LIRA, O. C. A Mata Atlântica do Nordeste. Recife: AMANE (Associação para Proteção da Mata Atlântica do Nordeste). 2006, 17 p.

TALARICO, F.; BRANDMAYR, P.; GIULIANINI, P.G.; IETTO, F.; NACCARATO, A.; PERROTTA, E.; TAGARELLI, A.; GIGLIO, A. Effects of metal pollution on survival and physiological responses in Carabus (Chaetocarabus) lefebvrei (Coleoptera, Carabidae). European Journal of Soil Biology, v. 9, p. 80-89, 2014.

PAYLISS, B. J., HASSANIN, M., PRENNER, E. J. The structural and functional effects of $\mathrm{Hg}(\mathrm{II})$ and $\mathrm{Cd}(\mathrm{II})$ on lipid model systems and human erythrocytes: A review. Chemistry and Physics of Lipids, v. 193, p. 36-51, 2015.

YU, C., TING-MEI, X., PING ZHENG, Y., HUI XU, D. Zn(II)-curcumin protects against hemorheological alterations, oxidative stress and liver injury in a rat model of acute alcoholism. Environmental Toxicology and Pharmacology, v. 37, p. 729-737, 2014. 